Research Paper

\title{
High serum CCL1 8 predicts a poor prognosis in patients with laryngeal squamous cell carcinoma
}

Juncheng Wang 1,2, Yuexiang Qin 1,2,3, Gangcai Zhu 4, Donghai Huang 1,2, Ming Wei 1,2, Guo Li 1,2, Li She 1,2, Diekuo Zhang 1,2, Gang Wang 1,2, Xiyu Chen 1,2, Zhe Shen 1,2, Yuanzheng Qiu 1,2, Yunyun Wang 1,2, Haolei Tan ${ }^{5}$, Pingqing Tan ${ }^{5}$, Jie Chen ${ }^{5}$, Xin Zhang 1,2, ${ }^{,}$Yong Liu ${ }^{1,2}{ }^{\varpi}$

1. Department of Otolaryngology Head and Neck Surgery, Xiangya Hospital, Central South University, 87 Xiangya Road, Changsha, Hunan 410008, People's Republic of China.

2. Otolaryngology Major Disease Research Key Laboratory of Hunan Province, 87 Xiangya Road, Changsha, Hunan 410008, People's Republic of China.

3. Department of Health Management, the Third Xiangya Hospital, Central South University, 138 Tongzipo Road, Changsha Hunan 410013, People's Republic of China.

4. Department of Otolaryngology Head and Neck Surgery, the Second Xiangya Hospital, Central South University, 139 Renmin Road, Changsha, Hunan 410011, People's Republic of China.

5. Department of Head and Neck Surgery, Hunan Cancer Hospital, The Affiliated Tumor Hospital of Xiangya Medical School, Central South University, 283 Tongzipo Road, Changsha, Hunan 410013, People's Republic of China.

*These authors contributed equally to this work.

$\square$ Corresponding author: Yong Liu (liuyongent@csu.edu.cn) and Xin Zhang (xinzhang@csu.edu.cn).

( ) The author(s). This is an open access article distributed under the terms of the Creative Commons Attribution License (https://creativecommons.org/licenses/by/4.0/). See http://ivyspring.com/terms for full terms and conditions.

Received: 2019.06.11; Accepted: 2019.09.30; Published: 2019.11.17

\begin{abstract}
CCL18 is a cytokine secreted by M2 type tumor associated macrophages, which frequently over-expressed in diverse human cancers. However, the clinical significance of serum CCL18 in patients with laryngeal squamous cell carcinoma (LSCC) remains unknown. In this study, serum CCL18 was initially quantified by enzyme-linked immunosorbent assay (ELISA) in 146 patients with LSCC, 25 patients with precancerous lesions and 72 healthy volunteers. In addition, the correlations between serum CCL18 and clinicopathological parameters were analyzed. Our data revealed that serum CCL18 was obviously increased in patients with LSCC. Moreover, serum CCL18 level was significantly associated with primary tumor site (Glottic vs Others), T classification ( $T 1+T 2$ vs $\mathrm{T3}+\mathrm{T4}$ ), clinical stage $(I+I I$ vs $I I I+I V)$ and lymph node metastasis (N0 vs $\mathrm{N}+$ ). Survival analysis demonstrated that patients with high serum CCL18 displayed a shorter survival time than those in patients with low serum CCL18. Importantly, serum CCL18 level and clinical stage were independent prognostic factors in patients with LSCC. Taken together, serum CCL18 could be used as a promising biomarker in patients with LSCC.
\end{abstract}

Key words: CCL18; LSCC; Prognosis; Tumor associated Macrophage.

\section{Introduction}

Laryngeal squamous cell carcinoma (LSCC), as one of the head and neck squamous cell carcinoma (HNSCC), has the highest rate of voice-related disabilities and a poor mortality [1]. Therapy for patients with LSCC including surgery, radiotherapy and chemotherapy, which are used either alone or in combination has been improved over the last few decades [2, 3]. Unfortunately, the life quality and survival status for patients with LSCC are far from satisfactory $[4,5]$. Cancer initiation and progression are closely linked with the aberrant expression and function of endogenous oncogenes or suppressors in cancer cells themselves, the remodeling of tumor microenvironment (TME) and the mutual interaction between cancer cells and the components of TME [6].

Tumor associated macrophages (TAMs) is a critical component in TME, which originates from circulating blood monocyte and then differentiates into M1 or M2 type TAMs once entry into tumor [7]. TAMs especially M2 type in primary tumor site, 
stimulates angiogenesis, enhances metastasis and also represses anti-tumor immune response, which indicates the protumoral role of TAMs in TME [8-10]. Therapeutic success in targeting these protumoral roles in preclinical models and in early clinical trials suggests that TAMs are attractive targets as part of immunotherapy in cancer treatment [11].

Cytokines and chemokines secreted by TAMs participate in the malignant progression of cancers. Among these secretions, CCL18 is an important cytokine that derived from M2 type TAMs [12]. CCL18 has been identified to function importantly in the invasion and metastasis of human cancers including head and neck cancer [13-16]. High enriched CCL18 was tightly related to poor prognosis in multiple solid cancers, such as pancreatic ductal adenocarcinoma [17, 18], ovarian cancer [19], gallbladder carcinoma [20] and gastric cancer [21] etc. However, the clinical significance and especially prognostic value of serum CCL18 in patients with LSCC remains unclear.

In this study, we evaluated the levels of serum CCL18 by Enzyme-linked immunosorbent assay (ELISA) and investigated the association of CCL18 expression with the clinicopathological variables in 146 patients with LSCC. Our results reveal that serum CCL18 is increased in patients with LSCC, which is linked with diverse malignant phenotypes of LSCC including advanced clinical stages, lymph node metastasis etc. More importantly, examination of serum CCL18 provides useful prognostic information for patients with LSCC.

\section{Material and methods}

\section{Subjects}

Blood serum samples from 146 patients with LSCC, whose age was ranged from 31 to 80 years (mean $50.6 \pm 12.0$ years), were collected prior to treatment. As controls, blood samples from 25 patients with precancerous lesions and 72 healthy volunteers were also collected. All the 146 patients with LSCC received standard treatment between September 2012 and April 2014 in the Department of Otolaryngology head and neck surgery at Xiangya Hospital, Central South University, Changsha, China. Patients enrolled in our study had to follow these inclusion criteria: Primary squamous cell carcinoma without other malignancies; no history of previous radiotherapy and chemotherapy. As showed in Table 1, the clinical data including age, gender, primary tumor site, T classification, clinical stage, histological grade and lymph node metastasis were recorded in detail. No other cancer history was recorded for them. Pathological tumor-node-metastasis (TNM) stage was determined according to the 7th American Joint Committee on Cancer staging system. The protocol was approved by the Medical Research Ethics Committee of Xiangya Hospital, Central South University, Hunan, China.

Table 1. The relationship between serum CCL18 and clinicopathological variables in patients with LSCC.

\begin{tabular}{llllll}
\hline Parameters & Number & \multicolumn{2}{c}{ Serum CCL18 levels } & P-value \\
\cline { 3 - 5 } & & Q25 & Q50 & Q75 & \\
\hline Age & 72 & 24640.85 & 34053.28 & 47889.54 & 0.425 \\
$>56$ & 74 & 26698.96 & 37512.85 & 54826.48 & \\
$<=56$ & & & & & \\
Gender & 7 & 37298.59 & 46965.44 & 58073.71 & 0.116 \\
Female & 139 & 25589.64 & 34880.33 & 49652.10 & \\
Male & & & & & \\
Primary tumor site & & 22018.72 & 32587.97 & 44815.06 & $<\mathbf{0 . 0 1}$ \\
Glottic & 93 & 31658.10 & 40500.12 & 73557.88 & \\
Others & 53 & & & & \\
Lymph node status & & & & \\
N0 & 94 & 22158.37 & 30799.68 & 38760.60 & $<\mathbf{0 1}$ \\
N+ & 52 & 36791.40 & 51178.05 & 89003.81 & \\
T classification & & & & & \\
T1+T2 & 72 & 21840.36 & 29029.14 & 38884.02 & $<0.01$ \\
T3+T4 & 74 & 32077.89 & 40941.73 & 61300.08 & \\
Clinical stage & & & & & \\
I+II & 57 & 20369.22 & 26594.64 & 34894.13 & $<0.01$ \\
III+ IV & 89 & 32424.43 & 42258.68 & 69677.39 & \\
Histological grade & & & & & \\
G1 & 15 & 26333.71 & 29323.18 & 49652.10 & 0.404 \\
G2+G3 & 131 & 25861.68 & 37216.18 & 52704.01 & \\
\hline
\end{tabular}

\section{Enzyme-linked immunosorbent assay (ELISA) for serum CCL18}

All blood samples were collected before initial treatment. To avoid the effects of diet on the level of serum CCL18, all participants were told to fast more than 8 hours, and their blood samples were collected in the morning between 7 a.m. and 10 a.m. by venipuncture (BD Vacutainer Plus). Blood samples were allowed to clot at room temperature followed by centrifuging at $3000 \mathrm{rpm}$ for $15 \mathrm{~min}$, and then tested within 2 hours. Samples with hemolysis were excluded from the study. Serum CCL18 was determined by an ELISA commercial kit (R\&D Systems, MN, USA) according to the manufacturer's instructions. Each experiment was performed in triplicate.

\section{Data collection and follow up}

According to the contact information provided by patients, the following methods were used for following up: outpatient consultation, telephone and letter visits. Survival time of patients with LSCC was calculated from the date of surgery. Patient death, loss of follow-up or the last follow-up time were taken as the end point of the follow-up. 133 patients (91.1\%) with LSCC accepted postoperational following up and detailed information was recorded, 13 patients failed to follow up due to changes in phone numbers 
and/or home address.

\section{Statistical analysis}

The statistical analysis was performed using SPSS (version 22.0, SPSS Inc., Chicago, IL, USA). The Mann-Whitney U-test was used to analyze the differences between groups. A $P$ value of $<0.05$ was considered as statistical significance.

\section{Results}

\section{Serum CCL1 8 levels in patients with LSCC and healthy controls}

To determine whether serum CCL18 were relevant to LSCC, peripheral blood samples were obtained from 146 patients with LSCC, 25 patients with precancerous lesions and 72 healthy controls. Our data showed that serum content value of CCL18 in patients with LSCC was $44086 \pm 2408 \mathrm{pg} / \mathrm{mL}$, while in those healthy individuals was $25588 \pm 1938 \mathrm{pg} / \mathrm{mL}$ and in patients with precancerous lesions was $28945 \pm$ $2851 \mathrm{pg} / \mathrm{mL}$. The mean concentration of serum CCL18 in patients with LSCC was dramatically higher than that in patients with precancerous lesions and healthy individuals (Fig.1; $P=0.0118$ ), which no difference existed between patients with precancerous lesions and healthy volunteers $(P=0.3659)$.

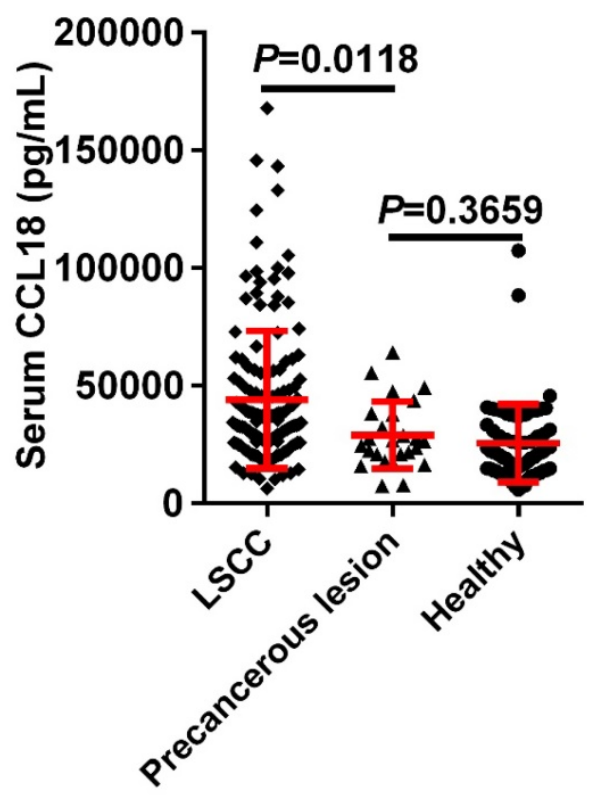

Figure 1. Serum CCL18 in patients with LSCC, patients with precancerous lesions and healthy volunteers was quantified by ELISA. Each dot represents one sample in each group.

\section{Clinical associations of serum CCL18 in patients with LSCC}

Since serum CCL18 was elevated in patients with LSCC, we further asked whether serum CCL18 levels correlated with the clinicopathological parameters in patients with LSCC. As illustrated in Table 1, serum CCL18 level was positively associated with primary tumor site (Glottic vs. Others; $P<0.001$ ), tumor classification (T1+T2 vs. T3+T4; $P<0.001)$, clinical stage (I+II vs. III+IV; $P<0.001$ ) and lymph node metastasis (N0 vs. N+; $P<0.001$ ). However, there was no relationship between serum CCL18 and other parameters including age $(P=0.425)$, gender $(P$ $=0.116)$ and histological grade $(P=0.404)$. Taken together, these data suggests that serum CCL18 level is a potentially valuable biomarker in the progression of LSCC.

\section{High serum CCL1 8 predicts a worse prognosis in patients with LSCC}

In our study, a total of 146 patients with LSCC were enrolled, in which 133 patients (91.1\%) with intact clinicopathological information were used for further survival analysis. Among these 133 patients, 41 patients $(30.8 \%)$ died at different time after surgery, and 54 patients $(40.6 \%)$ experienced local recurrence by fibrolaryngoscopy, CT and MRI. To investigate the potential association between serum CCL18 level and prognosis, patients were divided into two groups including high serum CCL18 $(\mathrm{n}=66)$ and low serum CCL18 ( $\mathrm{n}=67)$, in which serum CCL18 level was greater than or lower than the median value in our patient cohort. Therefore, Kaplan-meier method and log-rank test were then used to calculate the 5-year overall survival rate $(60.61 \%$ vs. $77.61 \% ; P=0.029$; Fig.2) in these two groups. Patients with high serum CCL18 had a shorter overall survival time (median survival time $40.8 \pm 9.7$ months vs. $53.9 \pm 5.5$ months). Collectively, these data reveals that serum CCL18 could be used as a predictor for the prognosis of patients with LSCC.

\section{Serum CCL1 8 level was an independent factor in patients with LSCC}

Eight clinical parameters including age, gender, primary tumor site, histopathological grade, $\mathrm{T}$ staging, presence or absence of lymph node metastasis, clinical staging and serum CCL18 level were initially enrolled in univariate analysis. Univariate Cox regression analyses initially indicated that primary tumor site, $\mathrm{T}$ staging, clinical stage, lymph node metastasis and serum CCL18 level were significantly associated with the overall survival in patients with LSCC (All $P<0.05$; Table 2). However, only clinical stage $(P=0.006$; Table 2$)$ and serum CCL18 level $(P=0.001$; Table 2$)$ were independent factors affecting the prognosis in patients with LSCC. The relative risk (RR) of CCL18 level in serum on the overall survival rate was 1.538 , with a $95 \%$ confidence interval of [0.926-3.017]. 


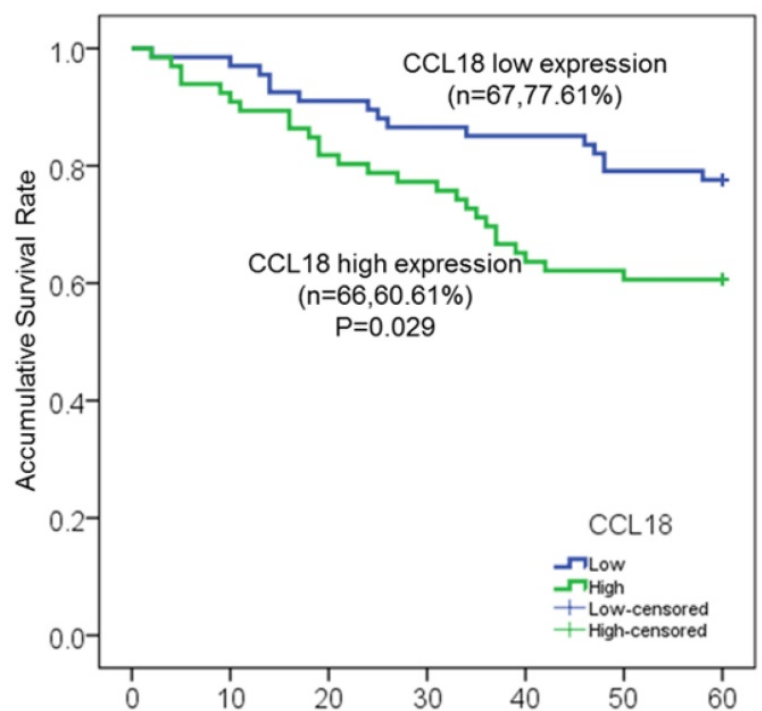

Figure 2. Kaplan-Meier survival curves of serum CCL18 high expression and low expression in patients with LSCC.

Table 2. Univariate and multivariate Cox analysis of variables considered for overall survival in patients with LSCC.

\begin{tabular}{|c|c|c|}
\hline \multirow[t]{2}{*}{ Parameters } & Overall Survival & $P$-value \\
\hline & \multicolumn{2}{|l|}{ Relative risk $(95 \% \mathrm{CI})$} \\
\hline \multicolumn{3}{|l|}{ Univariate } \\
\hline Gender & $2.290(0.315-16.661)$ & 0.413 \\
\hline Age & $1.487(0.802-2.755)$ & 0.208 \\
\hline Primary tumor site & $2.071(1.122-3.823)$ & 0.020 \\
\hline T classification & $2.626(1.339-5.151)$ & 0.005 \\
\hline Lymph node status & $2.987(1.601-5.572)$ & 0.001 \\
\hline Clinical stage & $5.674(2.224-14.476)$ & $<0.01$ \\
\hline Histological grade & $0.707(0.297-1.681)$ & 0.432 \\
\hline Serum CCL18 & $3.212(2.091-4.732)$ & $<0.01$ \\
\hline \multicolumn{3}{|l|}{ Multivariate } \\
\hline Serum CCL18 & $1.538(0.926-3.017)$ & 0.001 \\
\hline Clinical stage & $3.929(1.477-10.452)$ & 0.006 \\
\hline
\end{tabular}

\section{Discussion}

TME is largely orchestrated by inflammatory cells, is an indispensable participant in the neoplastic process, which favors malignant cell proliferation, survival, metastasis and resistance to cancer therapy etc [22]. M2-type TAMs, as one of the most critical components in TME, play a key role in the crosstalk between cancer cells and their surrounding TME [8]. M2 type TAMs actively participate in diverse malignant processes such as tumor extracellular matrix remodeling, immune suppression and angiogenesis, which are accelerators that promotes cancer metastasis and resistance to therapy $[9,11,23]$.

In our study, we found that CCL18, as a representative C-C cytokine, was overexpressed in the serum of patients with LSCC. Clinical significance analyses revealed that serum CCL18 level was closely linked with primary tumor site, tumor classification, clinical stage, lymph node metastasis and recurrence in patients with LSCC. In contrast, age, gender and histological grade did not correlate to the serum CCL18 level. More importantly, high serum CCL18 level was able to predict the 5-year overall survival time, which was an independent factor associated with the overall survival in patients with LSCC. Therefore, CCL18 is a potentially valuable serum biomarker for the progression and prognosis in patients with LSCC.

CCL18 may directly or indirectly promote tumor angiogenesis, suppress anti-cancer immune response and reshape TME, leading to malignant progression in diverse solid human cancers. CCL18 is mainly expressed and spontaneously secreted by cancer cells and stoma cells including monocytes, macrophages and immature dendritic cells etc. CCL18 secreted from M2-type TAMs is increased not only in chronic inflammations [24], fibrotic diseases [25, 26] and white adipose tissue dysfunction [27], but also in various types of neoplastic diseases. Song's team determined that serum CCL18 was obviously elevated in patients with breast carcinoma, and high CCL18 in the serum was linked with lymph node metastasis and worse histopathological typing, which was an independent influencing factor in the prognosis of patients with breast cancer [12]. Yuan etc reported that mean serum CCL18 levels elevated in epithelial ovarian cancer (EOC), which predicted a worse survival status in patients with EOC [19]. Similar results were also observed in pancreatic cancer [18], lung cancer [15]. In concert with the above available evidence, our study for the first time confirm the clinical significance of serum CCL18 in patients with LSCC, which highlights that serum CCL18 is a valuable biomarker in diverse human cancers. This founding underlines the importance of less-invasive assay of serum CCL18 in clinical management setting, which is more superior to other cancer biomarkers assayed via immunohistochemistry or $\mathrm{qPCR}$ in cancer tissue biopsy or surgery.

Although our study only confirms the clinical significance of serum CCL18 in patients with LSCC, CCL18 is also reported to be an efficient molecular target in TME to repress cancer progression. In one respect, CCL18 in TME binds to its receptors (PITPNM3 [12], CCR6 [28] and CCR8 [29-31]) on the membrane of tumor cells, activates multiple carcinogenesis associated signaling pathways including NF-KB, PI3K/Akt, Wnt/ $\beta$-catenin and mTOR etc, which in turn accelerates the progression of diverse human cancers including head and neck cancer $[13,14]$. On the other hand, CCL18 as a secreted cytokine also remodels TME to promote cancer progression. For example, CCL18 represses the 
anti-cancer immune response by recruiting regulatory $\mathrm{T}$ (Treg) cells and induce an immune suppression environment that finally leads to suppress deleterious inflammation and cancer progression [32-35]. Therefore, cytokine CCL18 exert its function via influencing both cancer cells and their surrounding TME.

In conclusion, our study reveals that serum CCL18 is increased in patients with LSCC, which is also linked with diverse malignant phenotypes of LSCC including advanced clinical stages, lymph node metastasis etc. More importantly, examination of serum CCL18 provides useful prognostic information for patients with LSCC. However, how CCL18 participates in the malignant progression of LSCC requires further verification both in vitro and in vivo.

\section{Acknowledgements}

This study was supported by the National Natural Science Foundation of China (Nos.81974424, 81874133, 81772903, 81773243, 81602389 and 81602684), the National Key Research and Development Program of China (No.2016YFC0 902003), the Natural Science Foundation of Hunan Province (Nos.2018JJ2630, 2017JJ3456, 2017JJ3488, 2019JJ50944 and 2019JJ40481), Project of Hunan Health Commission (No.B2019165), the Huxiang Young Talent Project (No.2018RS3024) and the Xiangya Hospital-Sinobioway Clinic and rehabilitation Research Foundation (No.XYWM 2015123).

\section{Competing Interests}

The authors have declared that no competing interest exists.

\section{References}

1. Siegel RL, Miller KD, Jemal A. Cancer statistics, 2016. CA Cancer J Clin. 2016; 66: 7-30.

2. Chawla S, Carney AS. Organ preservation surgery for laryngeal cancer. Head Neck Oncol. 2009; 1: 12.

3. Zhang C, Zhu M, Chen D, Chen S, Zheng H. Organ preservation surgery for patients with T4a laryngeal cancer. Eur Arch Otorhinolaryngol. 2018; 275: 1601-6

4. Forastiere AA, Ismaila N, Lewin JS, Nathan CA, Adelstein DJ, Eisbruch A, et al. Use of Larynx-Preservation Strategies in the Treatment of Laryngeal Cancer: American Society of Clinical Oncology Clinical Practice Guideline Update. J Clin Oncol. 2018; 36: 1143-69.

5. Steuer CE, El-Deiry M, Parks JR, Higgins KA, Saba NF. An update on larynx cancer. CA Cancer J Clin. 2017; 67: 31-50.

6. Wellenstein MD, de Visser KE. Cancer-Cell-Intrinsic Mechanisms Shaping the Tumor Immune Landscape. Immunity. 2018; 48: 399-416.

7. Franklin RA, Liao W, Sarkar A, Kim MV, Bivona MR, Liu K, et al. The cellular and molecular origin of tumor-associated macrophages. Science. 2014; 344: 921-5.

8. Qian BZ, Pollard JW. Macrophage diversity enhances tumor progression and metastasis. Cell. 2010; 141: 39-51.

9. Jiang C, Yuan F, Wang J, Wu L. Oral squamous cell carcinoma suppressed antitumor immunity through induction of PD-L1 expression on tumor-associated macrophages. Immunobiology. 2017; 222: 651-7.

10. Lian $G$, Chen $S$, Ouyang M, Li F, Chen L, Yang J. Colon Cancer Cell Secretes EGF to Promote M2 Polarization of TAM Through EGFR/PI3K/AKT/mTOR Pathway. Technol Cancer Res Treat. 2019; 18: 1533033819849068.

11. Noy R, Pollard JW. Tumor-associated macrophages: from mechanisms to therapy. Immunity. 2014; 41: 49-61.
12. Chen J, Yao Y, Gong C, Yu F, Su S, Chen J, et al. CCL18 from tumor-associated macrophages promotes breast cancer metastasis via PITPNM3. Cancer Cell. 2011; 19: 541-55.

13. She L, Qin Y, Wang J, Liu C, Zhu G, Li G, et al. Tumor-associated macrophages derived CCL18 promotes metastasis in squamous cell carcinoma of the head and neck. Cancer Cell Int. 2018; 18: 120.

14. Oin $\mathrm{Y}$, Wang J, Zhu G, Li G, Tan $\mathrm{H}$, Chen $\mathrm{C}$, et al. CCL18 promotes the metastasis of squamous cell carcinoma of the head and neck through MTDH-NF-kappaB signalling pathway. J Cell Mol Med. 2019; 23: 2689-701.

15. Shi L, Zhang B, Sun X, Zhang X, Lv S, Li H, et al. CC chemokine ligand 18(CCL18) promotes migration and invasion of lung cancer cells by binding to Nir1 through Nir1-ELMO1/DOC180 signaling pathway. Mol Carcinog. 2016; 55: 2051-62.

16. Lin L, Chen YS, Yao YD, Chen JQ, Chen JN, Huang SY, et al. CCL18 from tumor-associated macrophages promotes angiogenesis in breast cancer. Oncotarget. 2015; 6: 34758-73.

17. Ye $\mathrm{H}$, Zhou $\mathrm{O}$, Zheng $\mathrm{S}$, Li G, Lin $\mathrm{Q}$, Wei $\mathrm{L}$, et al. Tumor-associated macrophages promote progression and the Warburg effect via CCL18/NF-kB/VCAM-1 pathway in pancreatic ductal adenocarcinoma. Cell Death Dis. 2018; 9: 453.

18. Meng F, Li W, Li C, Gao Z, Guo K, Song S. CCL18 promotes epithelial-mesenchymal transition, invasion and migration of pancreatic cancer cells in pancreatic ductal adenocarcinoma. Int J Oncol. 2015; 46: 1109-20.

19. Yuan L, Wan J, Huang C, Liang J, Liu M, Yue C, et al. Evaluation of serum CCL18 as a potential biomarker for ovarian cancer. Cancer Biomark. 2017; 21: 97-104.

20. Zhou Z, Peng Y, Wu X, Meng S, Yu W, Zhao J, et al. CCL18 secreted from M2 macrophages promotes migration and invasion via the PI3K/Akt pathway in gallbladder cancer. Cell Oncol (Dordr). 2019; 42: 81-92.

21. Wu J, Liu X, Wang Y. Predictive value of preoperative serum CCL2, CCL18, and VEGF for the patients with gastric cancer. BMC Clin Pathol. 2013; 13: 15.

22. Lim B, Woodward WA, Wang X, Reuben JM, Ueno NT. Inflammatory breast cancer biology: the tumour microenvironment is key. Nat Rev Cancer. 2018; 18: 485-99.

23. Liu H, Yang L, Qi M, Zhang J. NFAT1 enhances the effects of tumor-associated macrophages on promoting malignant melanoma growth and metastasis. Biosci Rep. 2018; 38.

24. Kollert F, Binder M, Probst C, Uhl M, Zirlik A, Kayser G, et al. CCL18 potential biomarker of fibroinflammatory activity in chronic periaortitis. J Rheumatol. 2012; 39: 1407-12.

25. Prasse A, Pechkovsky DV, Toews GB, Schafer M, Eggeling S, Ludwig C, et al. CCL18 as an indicator of pulmonary fibrotic activity in idiopathic interstitial pneumonias and systemic sclerosis. Arthritis Rheum. 2007; 56: 1685-93.

26. Ossorio M, Martinez V, Bajo MA, Del Peso G, Castro MJ, Romero S, et al. Prominent Levels of the Profibrotic Chemokine CCL18 during Peritonitis: In vitro Downregulation by Vitamin D Receptor Agonists. Biomed Res Int. 2018; 2018: 6415892.

27. Eriksson Hogling D, Petrus P, Gao H, Backdahl J, Dahlman I, Laurencikiene I, et al. Adipose and Circulating CCL18 Levels Associate With Metabolic Risk Factors in Women. J Clin Endocrinol Metab. 2016; 101: 4021-9.

28. Luzina IG, Atamas SP. CCR6 is not necessary for functional effects of human CCL18 in a mouse model. Fibrogenesis Tissue Repair. 2012; 5: 2

29. Liu X, Xu X, Deng W, Huang M, Wu Y, Zhou Z, et al. CCL18 enhances migration, invasion and EMT by binding CCR8 in bladder cancer cells. Mol Med Rep. 2019; 19: 1678-86.

30. Berenguer J, Lagerweij T, Zhao XW, Dusoswa S, van der Stoop P, Westerman $\mathrm{B}$, et al. Glycosylated extracellular vesicles released by glioblastoma cells are decorated by CCL18 allowing for cellular uptake via chemokine receptor CCR8. J Extracell Vesicles. 2018; 7: 1446660.

31. Islam SA, Ling MF, Leung J, Shreffler WG, Luster AD. Identification of human CCR8 as a CCL18 receptor. J Exp Med. 2013; 210: 1889-98.

32. Azzaoui I, Yahia SA, Chang Y, Vorng H, Morales O, Fan Y, et al. CCL18 differentiates dendritic cells in tolerogenic cells able to prime regulatory $\mathrm{T}$ cells in healthy subjects. Blood. 2011; 118: 3549-58.

33. Chang Y, de Nadai P, Azzaoui I, Morales O, Delhem N, Vorng $\mathrm{H}$, et al. The chemokine CCL18 generates adaptive regulatory $\mathrm{T}$ cells from memory CD4+ T cells of healthy but not allergic subjects. FASEB J. 2010; 24: 5063-72.

34. Chenivesse C, Chang Y, Azzaoui I, Ait Yahia S, Morales O, Ple C, et al. Pulmonary CCL18 recruits human regulatory T cells. J Immunol. 2012; 189: 128-37.

35. Sun Z, Du C, Xu P, Miao C. Surgical trauma-induced CCL18 promotes recruitment of regulatory $\mathrm{T}$ cells and colon cancer progression. J Cell Physiol. 2019; $234:$ 4608-16. 